\title{
Sonoelastography of the Median Nerve in Carpal Tunnel Syndrome in Correlation with Nerve Conduction Studies
}

D.R.Lasheen, S.M.Abdelmoneam, A.Y.El Shambaky and M.Y.Mahgoub

Rheumatology and Rehabilitation, Dept., Faculty of Medicine, Benha Univ., Benha, Egypt

E-Mail: Doaa.reyad@fmed.bu.edu.eg

\begin{abstract}
Sonoelastography is a novel ultrasound (US) tool of portraying the strain properties of biological tissue. The carpal tunnel syndrome (CTS) may cause structural and elastic distortion of the median nerve (MN). We wanted to see how Bmode ultrasound and strain elastography differed between the CTS patient population and healthy individuals, as well as how they correlated with nerve conduction investigations (NCS). Methods: There were 100 wrists in this prospective study from 57 people with clinically confirmed CTS, and 110 wrists in this study from 55 healthy, non-diseased individuals. Patients with CTS had their neck circumference measured (NCS). The CSA of the MN at the wrist, the wrist-to-forearm ratio (WFR), and the flattening ratio (FR) were measured, and the MN's echogenicity and mobility were evaluated, in both the patient and control groups. Strain elastography was used to assess the MN's elasticity. The $\mathrm{MN}$ at the wrist was significantly stiffer in CTS patients than in healthy controls ( $\mathrm{p} 0.001)$. Compared to the control group, the MN CSA-D, WFR echogenicity, and mobility of the CTS participants were all statistically substantially different (p0.001). This study found a statistically significant association between the elastic ratio and the MN distal motor delay and a statistically significant correlation between the elastic ratio and the sensory amplitude and conduction velocity. When it comes to NCS neurophysiology, the MN elastic ratio was shown to have a positive connection $(\mathrm{r}=0.252$, $\mathrm{p}-0.011)$ with the neurophysiological grades. Sonoelastography in CTS demonstrated strong relationships with NCSs. Using sonoelastography in CTS was shown to be both useful and practicable in this investigation.
\end{abstract}

Key words: Strain elastography, carpal tunnel syndrome, neuromuscular ultrasound, nerve conduction studies.

\section{Introduction}

Neuropathy induced by the entrapment of the median nerve in the carpal tunnel is known as carpal tunnel syndrome (CTS). Entrapment neuropathies account for $90 \%$ of all entrapment neuropathies, although there is no agreed criteria for diagnosing them. Using the patient's medical history, physical exam findings, and any positive results from provocative testing, a doctor may make this diagnosis. [2]

CTS is diagnosed with the use of nerve conduction studies (NCS), which are used to assess the severity of the condition. NCSs, however, have a number of drawbacks that have prompted researchers to seek for an alternative method that is less intrusive and more convenient. 4] Currently, ultrasonography (US) is effective in supporting the diagnosis of CTS, with sensitivity and specificity reported to be between 57$97.9 \%$ and $51-100 \%$. There are several reasons why US is a useful technique, such as its inexpensive cost; short study time; little invasiveness; immediate results; and dynamic imaging. Ultrasound may be used to diagnose secondary CTS and reveal anatomical abnormalities, such as a persisting median artery or bifurcated $\mathrm{MN}$, as well as space-occupying lesions such as tenosynovitis and cysts, in the afflicted MN by direct viewing. Anatomical measurements of the MN's cross-sectional area (CSA) are most often employed in the United States to determine whether or not someone has carpal tunnel syndrome (CTS). Sonoelastography, a novel ultrasonographic feature of nerves, has been evaluated in certain studies finding increased stiffness of nerves affected by neuropathy [7]. In the field of elastography, strain and shear wave elastography are the two basic types. Strain elastography is a technique for determining the stiffness of visible objects by measuring tissue deformation under pressure. MN structural and elastic deformation may result from CTS.

Patients with CTS and healthy controls were compared to see how B-mode ultrasound and strain elastography performed, as well as to see whether there were any relationships between the two. NCSs.

\section{Subjects and methods}

This prospective study included 100 wrists of 57 patients clinically diagnosed as CTS according to the American Academy of Neurology Clinical Diagnostic Criteria of CTS [1]. All of the participants were drawn from the Rheumatology, Rehabilitation, and Physical Medicine department's EMG lab and outpatient clinic. An additional control group included 110 wrists from 55 otherwise healthy people of similar age and gender. The study was approved by the ethical committee of the Faculty of Medicine, Benha University. An informed consent was procured from all patients and controls.

Inclusion criteria:

- Patients aged 20-60 are eligible for participation.

- Patients fulfilling the clinical criteria for idiopathic CTS.

\section{Exclusion criteria}

- Patients younger than 20 or older than 60.

- History of neurological disorders that may produce numbness or paresthesia in the hand such as ulnar neuropathy, radiculopathy, polyneuropathy, myelopathy, or stroke that is confirmed by clinical examination.

- Patients with underlying morbidity that may cause CTS e.g., chronic renal failure, uncontrolled 
diabetes, autoimmune diseases, other systemic disease, or use of contraceptive pills.

- CTS patients who had previously received corticosteroid injections or undergone surgery for the condition as determined by ultrasound imaging, patients with a Bifid median nerve (MN), persisting median artery, or any other structural variant of the MN.

- Compression of the MN by masses such as lipomas, hemangiomas, hematomas, fibromas, and ganglion cysts is a risk factor for CTS.

The same examiner conducted all of the patients' examinations on the same day. NCSs, B-mode US and US strain elastography were followed consecutively.

\section{Nerve conduction studies}

NCSs were performed using a Neurowerk EMG unit with a four-channel evoked potential/EMG measuring system (SIGMA Medizin-Technik GmbH Germany).NCSs were done according to the protocol recommended by the American Association of Electrodiagnostic Medicine.[10] It was done at room temperature.

Antidromic sensory median nerve and ulnar nerve conduction studies were recorded from digit II and digit $\mathrm{V}$, respectively. Motor $\mathrm{MN}$ conduction studies with stimulation at the wrist and elbow and motor ulnar nerve conduction studies with stimulation at the wrist, above and below the elbow were recorded, both including F-waves.

The study was considered highly suggestive of an isolated CTS if the median studies were abnormal, showing marked slowing across the wrist (prolonged distal motor and sensory latencies). If the median studies are completely normal or equivocal, the comparison tests were done. [10] $\square$ The severity of CTS was classified according to Bland's classification system for CTS (Bland, 2000) into six grades. [12]

\section{Neuromuscular Ultrasound (NMUS) Evaluation}

NMUS evaluation was performed using a linear high frequency 6-15 MHz probe on LOGIQ P9 (Korea) - ultrasound machine equipped with an elastography software. B-mode US was performed on the MN of patients and controls.

A standard protocol with optimization of scanning parameters was performed as suggested by Cartwright and Walker (2013). [11] All images were obtained with the subject laying supine on the bed, shoulders slightly abducted, elbows extended, and the forearms supinated. Wrists were evaluated in the neutral position with the palm up and the fingers semi-extended.The machine settings were optimized, a large amount of gel was added, the weight of the probe was applied without additional pressure. The entire course of the $\mathrm{MN}$ in the carpal tunnel was assessed in both the transverse and longitudinal planes for variations, anomalies and pathologies that would exclude them from the study.

For each wrist, the MN cross-sectional areas (CSA) at the wrist and forearm and the flattening ratio (FR) were obtained along with a subjective assessment of the MN echogenicity, and mobility. The CSA-D of the MN was measured at the proximal inlet of the carpal tunnel at the scaphoid-pisiform level (the distal wrist crease). The CSA-P was measured in the forearm by tracing the MN $12 \mathrm{~cm}$ proximally. The CSA of the $\mathrm{MN}$ at the wrist and forearm were measured automatically by using the continuous tracing method of the nerve circumference, excluding the hyperechoic epineurial rim (i.e., tracing the inner margin of the epineurium of MN).The Wrist/ forearm ratio (WFR) was calculated dividing the CSA-D by CSA-P value. The flattening ratio (FR) was defined as the ratio of the nerve transverse axis to the antero-posterior axis and was assessed at the level of the pisiform bone. The Echogenicity was reported as: "Normal" if multiple fascicles could be seen, "Slightly reduced" if there were 2 or fewer fascicles, or "Decreased" if there were no fascicles. The mobility was evaluated at the proximal inlet of the carpal tunnel. The subjects were asked to make a fist slowly, ensuring that the fingers were fully flexed at the MCP and IP joints while the US transducer was held still. The grade of $\mathrm{MN}$ mobility was assessed as following: Grade 1: minimal movement of the $\mathrm{MN}$ in all directions, Grade 2: the MN moving freely in the transverse plane, but not diving deep in the flexor tendons, and Grade 3: the MN descending deeply and surrounded by the flexor tendons. The grade of MN mobility was measured twice and the higher grade was selected for analysis. [9]

\section{Sonoelastography evaluation}

Sonoelastographic assessments of the MN were performed with the same US machine and transducer. Positioning of the subjects and machine settings were adapted as the same for NMUS evaluation. Elastographic evaluation was undertaken according to the procedure suggested by Tatar et. al (2016): [5] The probe is positioned perpendicularly to take correct and reproducible measurements. Generous amount of gel was applied to the wrist, then the weight of the probe was applied without additional pressure. $\mathrm{MN}$ was visualized in the axial plane at the entrance of the carpal tunnel between the scaphoid and pisiform, using the B-Mode US. The MN was kept in the center of the screen.

Strain elastography was obtained by light compressions applied by the US probe. The elastograms were constructed automatically using the same optimal settings throughout the study. The elastogram appeared within a rectangular window as a translucent color coded, real-time image superimposed on the B-mode image. The color code indicated the relative stiffness of the tissues within the region of interest (ROI) and ranged from red (soft) to blue (hard). Green and yellow colors indicated medium elasticity.

Sonoelastographic images were obtained by means of repeated light compressions with the probe on the MN in the sagittal plane at the level of the carpal tunnel inlet. The probe was compressed and decompressed with approximately the same force and 
duration. The measurements were done when more than half of the strain indicator was green. The screen was frozen at the decompression phase. The strain indicator indicated whether the displacement was sufficient to obtain local strains within the region of interest (ROI). Elliptical ROIs were drawn within the $\mathrm{MN}$ and again within the flexor carpi radialis (FCR), which was chosen as a reference tissue as it is outside the carpal tunnel. The ROI was drawn to be the largest ellipse that would fit entirely within the structures. The elastic ratio (ER) is the ratio of strain distribution in the two selected regions of interests (ROI). Strain indices (SI) were calculated by the device automatically, measurements were repeated 3 times, and the average strain ratio was recorded.

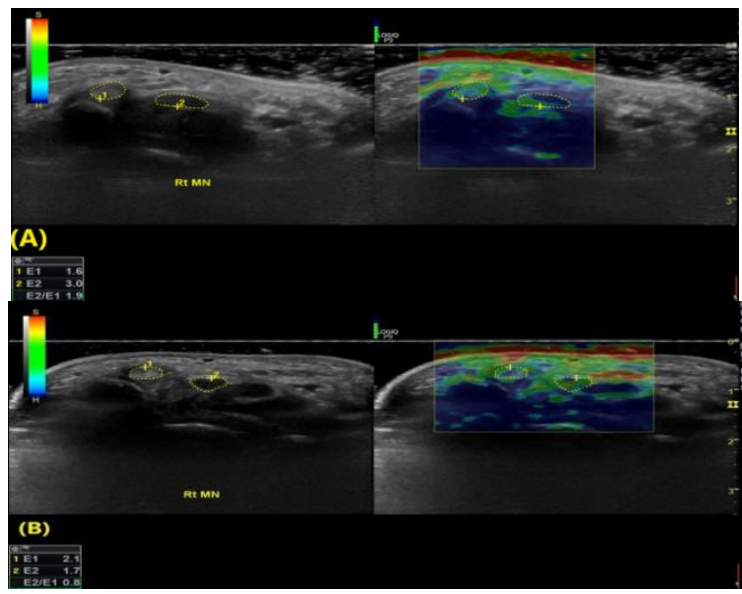

Fig. (1) B-mode ultrasonographic and sonoelastographic images of the median nerve in the transverse plane at carpal tunnel inlet. (A) 38-year-old female patient with mild carpal tunnel syndrome, (B) 25-year-old female healthy volunteer. The displayed color spectrum indicates stiffness of the tissues, ranging from red (soft) to blue (hard). ROI 1 was put along reference tendon (flexor carpi radialis tendon). ROI 2 was put along the median nerve at carpal tunnel inlet. (E1) Elasticity index of reference tissue, (E2) Elasticity index of median nerve, and (E2/E1) elasticity ratio of median nerve at the carpal tunnel inlet.

\subsection{Statistical analysis}

Collected data was revised, coded, tabulated using a Statistical Package for Social Science (IBM Corp. Released 2017. IBM SPSS Statistics for Windows, Version 25.0. Armonk, NY: IBM Corp.). The Kolmogorov-Smirnov test was used to test the normal distribution of data. The difference in the NMUS and elastography parameters between CTS patients and healthy controls were elucidated by the Student's t-test. Mann Whitney Test ( $U$ test) was used to assess the statistical significance of the difference of a nonparametric variable between two study groups. ChiSquare test was used to examine the relationship between two qualitative variables. Fisher's exact test was used to examine the relationship between two qualitative variables when the expected count is less than 5 in more than $20 \%$ of cells. Correlations between parameters were evaluated using the Pearson correlation coefficient. $\mathrm{P}$ value $<0.001$ was considered highly significant (HS) while a $\mathrm{P}$ value $>0.05$ was considered non-significant.

\section{Results}

Demographic and clinical data among the studied groups; Table (1).

The Body mass index was the only parameter that was statistically significantly different $(\mathrm{p}=0.037)$ between the studied groups. Among the patients, the reversed Phalen test was positive in all wrists (100\%) while the Tinel sign was positive in 76 wrists $(76 \%)$.

\section{Results of the electrodiagnostic studies}

In 49 wrists $(49 \%)$, the median routine NCS were completely normal. Comparative tests were done and found normal in 35 wrist $(35 \%)$ and positive in 14 wrists (14\%).

According to Bland's classification system for CTS (Bland, 2000),[12] the patients' wrists in our study were classified into six grades: normal $(35 \%)$, grade $1(14 \%)$, grade $2(6 \%)$, grade $3(28 \%)$, grade 4 $(6 \%)$, grade $5(8 \%)$ and grade $6(3 \%)$.

Neuromuscular ultrasound and Sonoelastographic findings in the studied groups

Table (2) summarizes the mean values and the comparisons between the cases and the control groups of each NMUS parameter and the elastic ratio.

Correlations of NMUS and sonoelastographic parameters with nerve conduction studies parameters in the CTS group; Table (3)

Regarding the sonoelastographic parameters, the ER showed a statistically significant positive correlation $(\mathrm{r}=, \mathrm{p}<0.05)$ with the distal motor latency $(\mathrm{r}=0.206, \mathrm{p}=0.040)$. The ER showed a statistically significant negative correlation $(p<0.05)$ with the sensory amplitude $(\mathrm{r}=-0.227, \mathrm{p}=0.023)$ and the sensory conduction velocity $(\mathrm{r}=-0.312, \mathrm{p}=0.002)$. There was a statistically significant positive correlation $(\mathrm{p}<0.05)$ between the $\mathrm{MN}$ elastic ratio and the neurophysiological grades of NCS $(r=0.252, p=0.011)$. 
Table (1) Comparisons between the cases and the control group regarding the demographic data.

\begin{tabular}{|c|c|c|c|c|c|}
\hline \multirow{3}{*}{ 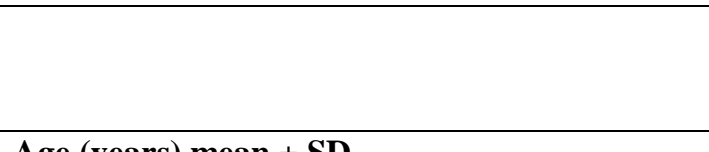 } & \multicolumn{4}{|c|}{ Subjects } & \multirow{3}{*}{$\begin{array}{c}\mathbf{p} \\
0.080\end{array}$} \\
\hline & \multicolumn{2}{|r|}{$\begin{array}{l}\text { Case } \\
\mathrm{n}=57\end{array}$} & \multicolumn{2}{|c|}{$\begin{array}{c}\text { Control } \\
\mathbf{n}=55\end{array}$} & \\
\hline & 39 & \pm 9.8 & 35.7 & \pm 10.3 & \\
\hline \multirow{2}{*}{ Sex; N, \% } & 3 & $5.3 \%$ & 7 & $12.7 \%$ & \multirow{2}{*}{0.199} \\
\hline & 54 & $94.7 \%$ & 48 & $87.3 \%$ & \\
\hline Weight $(\mathrm{Kg})$ mean \pm SD & 83.1 & \pm 13.8 & 79.1 & \pm 15.6 & 0.157 \\
\hline Height $(m)$ mean \pm SD & 1.61 & \pm 0.07 & 1.63 & \pm 0.08 & 0.146 \\
\hline $\mathrm{BMI}(\mathrm{Kg} / \mathrm{m} 2)$ mean $\pm \mathrm{SD}$ & 32.1 & \pm 5.0 & 29.9 & \pm 5.8 & $\mathbf{0 . 0 3 7}(\mathbf{S})$ \\
\hline Symptom's duration (months) median (range) & 12 & $(2-60)$ & - & - & - \\
\hline
\end{tabular}

SD: Standard deviation, N: number, p: Probability value, $\mathrm{p}<.05=$ significant $(\mathrm{S}), \mathrm{p}>.05=$ insignificant, $\mathrm{P}<0.001=$ high significant (HS), BMI: Body Mass Index

Table (2) Comparisons of the NMUS findings between the cases and the control group.

\begin{tabular}{|c|c|c|c|c|c|c|}
\hline \multirow{3}{*}{ Echogenicity: N, \% } & \multirow{5}{*}{$\begin{array}{l}\text { Normal } \\
\text { slightly reduced } \\
\text { Reduced }\end{array}$} & \multicolumn{4}{|c|}{ Wrists } & \multirow{5}{*}{$\begin{array}{c}\text { P } \\
<0.001 \\
\text { (HS) }\end{array}$} \\
\hline & & \multicolumn{2}{|c|}{$\begin{array}{c}\text { Case } \\
\mathrm{n}=100\end{array}$} & \multicolumn{2}{|c|}{$\begin{array}{c}\text { Control } \\
n=110\end{array}$} & \\
\hline & & 29 & $29 \%$ & 108 & $98 \%$ & \\
\hline & & 54 & $54 \%$ & 2 & $2 \%$ & \\
\hline & & 17 & $17 \%$ & 0 & $0 \%$ & \\
\hline Mobility grades: N, \% & 1 & 22 & $22 \%$ & 0 & $0 \%$ & \\
\hline & 2 & 48 & $48 \%$ & 12 & $11 \%$ & $<0.001$ \\
\hline & 3 & 30 & $30 \%$ & 98 & $89 \%$ & \\
\hline CSA-D $\left(\mathrm{mm}^{2}\right):$ mean \pm SD & & 14.6 & 4.6 & 9.0 & 1.9 & $\begin{array}{c}<0.001 \\
(\text { HS })\end{array}$ \\
\hline CSA-P $(\mathrm{mm}):$ mean \pm SD & & 6.7 & 2.1 & 7.3 & 2.3 & 0.263 \\
\hline WFR: median, range & & 2.12 & $0.87-5.66$ & 1.25 & $0.75-2.20$ & $\begin{array}{c}<0.001 \\
\text { (HS) }\end{array}$ \\
\hline FR: mean \pm SD & & 2.9 & 0.8 & 2.7 & 0.8 & 0.529 \\
\hline Elastic ratio: mean \pm SD & & 1.63 & 0.48 & 0.82 & 0.27 & $\begin{array}{c}<0.001 \\
(\mathrm{HS})\end{array}$ \\
\hline
\end{tabular}

NMUS, Neuromuscular ultrasound; CSA-D, cross sectional area distal at carpal tunnel; CSA-P, cross sectional area proximal at midforearm; WFR, Wrist/ forearm ratio; FR, Flattening ratio, SD: Standard deviation, N: number, P: Probability value, $\mathrm{P}<.05=$ significant $(\mathrm{S}), \mathrm{P}>.05=$ insignificant, $\mathrm{P}<0.001=$ high significant $(\mathrm{HS})$.

Table (3) Correlations between NMUS parameters with electrodiagnostic studies parameters in the CTS group.

\begin{tabular}{llcccccc}
\hline \multicolumn{1}{c}{ Parameters } & & CSA-D & CSA-P & WFR & Echogenicity & Mobility & FR \\
\hline Distal motor latency & $\mathbf{r}$ & 0.624 & 0.032 & 0.584 & 0.472 & -0.428 & 0.259 \\
Motor Amplitude & $\mathbf{p}$ & $<\mathbf{0 . 0 0 1}$ & 0.754 & $<\mathbf{0 . 0 0 1}$ & $<\mathbf{0 . 0 0 1}$ & $<\mathbf{0 . 0 0 1}$ & $\mathbf{0 . 0 0 9}$ \\
& $\mathbf{r}$ & -0.313 & 0.083 & -0.339 & -0.309 & 0.271 & -0.179 \\
Motor conduction & $\mathbf{p}$ & $\mathbf{0 . 0 0 2}$ & 0.411 & $\mathbf{0 . 0 0 1}$ & $\mathbf{0 . 0 0 2}$ & $\mathbf{0 . 0 0 6}$ & 0.076 \\
velocity & $\mathbf{r}$ & -0.357 & -0.082 & -0.294 & -0.289 & 0.285 & -0.054 \\
Sensory latency & $\mathbf{p}$ & $<\mathbf{0 . 0 0 1}$ & 0.420 & $\mathbf{0 . 0 0 3}$ & $\mathbf{0 . 0 0 3}$ & $\mathbf{0 . 0 0 4}$ & 0.597 \\
& $\mathbf{r}$ & -0.112 & -0.053 & -0.095 & 0.028 & 0.068 & -0.065 \\
Sensory Amplitude & $\mathbf{p}$ & 0.269 & 0.600 & 0.347 & 0.782 & 0.501 & 0.522 \\
& $\mathbf{r}$ & -0.531 & -0.116 & -0.453 & -0.366 & 0.393 & -0.258 \\
Sensory Conduction & $\mathbf{p}$ & $\mathbf{< 0 . 0 0 1}$ & 0.250 & $<\mathbf{0 . 0 0 1}$ & $<\mathbf{0 . 0 0 1}$ & $<\mathbf{0 . 0 0 1}$ & $\mathbf{0 . 0 0 9}$ \\
Velocity & $\mathbf{r}$ & -0.576 & -0.074 & -0.506 & -0.459 & 0.377 & -0.265 \\
Neurophysiological & $\mathbf{p}$ & $\mathbf{< 0 . 0 0 1}$ & 0.462 & $<\mathbf{0 . 0 0 1}$ & $<\mathbf{0 . 0 0 1}$ & $<\mathbf{0 . 0 0 1}$ & $\mathbf{0 . 0 0 8}$ \\
grade & $\mathbf{r}$ & 0.686 & 0.066 & 0.632 & 0.507 & -0.421 & 0.310 \\
\hline
\end{tabular}

NMUS, Neuromuscular ultrasound; CSA-D, cross sectional area distal at carpal tunnel; CSA-P, cross sectional area proximal at midforearm; WFR, Wrist/ forearm ratio; FR, Flattening ratio, p: Probability value, $p<.05=$ significant $(S), p$ $>.05=$ insignificant, $\mathrm{P}<0.001=$ high significant $(\mathrm{HS}), \mathrm{r}$ : Pearson correlation coefficient. 


\section{Discussion}

The benefits of ultrasound and the enhancement of its picture quality have led to a greater adaption of ultrasound investigations in various musculoskeletal disorders. CSA and WFR values were utilised to support the CTS diagnosis. [7] Using sonoelastography, researchers have found a new way to examine CTS. B-mode ultrasound and strain elastography were used in this research to evaluate their involvement in the treatment of patients with CTS, as well as to examine their connections with the National Center for Health Statistics.

Most typically, the CSA of MN at the proximal carpal tunnel is observed in diagnosis of CTS using US technology [13]. In comparison to healthy controls, we discovered a significantly larger MN CSA at the entrance of the carpal tunnel, as well as a reduction in echogenicity. Our findings were in line with those of earlier research [13-16], which showed that those with CTS had a higher MN CSA than healthy people. In addition, they found that the sonographic and electrodiagnostic results were in excellent agreement. $[17,18]$

When the $\mathrm{MN}$ is compressed, the blood-nerve barrier collapses, resulting in an elevated endoneurial fluid pressure, which causes nerve edema and swelling and further impairs the local blood flow in the affected area of the body. The CSA rises and the MN's echogenicity decreases as a result of this. [19]

The wrist/forearm ratio (WFR) is the second most often used criterion for measuring the MN. When compared to healthy volunteers, we discovered that the WFR was higher in CTS patients. Our findings were in line with those of earlier research. [14, 20 and 21] Hobson-Webb et al. (2008) reported that the WFR of 1.4 or more showed $100 \%$ sensitivity for detecting patients suffering from CTS.[20] On the other hand, some studies reported different results. [18, 22, 23]

The mechanical environment within the carpal tunnel affects the MN motion. Unlike the MN CSA, there has been a lack of consensus on the application of nerve mobility due to the complexity of dynamic analysis. [24] In our study, we found a restricted MN mobility in CTS patients compared to the controls. MN mobility correlated significantly with NCSs grading results. Similar results were reported in previous studies. [25, 26] Park et al., 2018 hypothesized that a reduced MN mobility in CTS may be related to fibrosis and adherence of the nerve to the flexor retinaculum. [25]

The flattening ratio (FR) of the $\mathrm{MN}$ was previously studied with controversial results. In our study, it was not found to be a useful discriminatory criterion in CTS patients that coincided with the results of Wong et al.(2002), Roll et al. (2011), and Chang et al., (2019). [27-29] Wong et al., 2002 suggested that the $\mathrm{MN}$ is a quite malleable structure, visibly changing in shape with movements of the flexor tendons resulting in the poor reliability of the FR measurements. [27] Our results were not consistent with those of Mallouhi et al. (2006), Gonzalez-Suarez et al. (2019) and Pertea et al. (2019). [23,30,31]

On sonoelastographic evaluation of the $\mathrm{MN}$ in CTS patients using strain elastography, we found that the $\mathrm{MN}$ at the wrist was highly significantly stiffer than in the healthy controls. The ER correlated significantly with the results of NCSs.

Our results are in parallel with previous studies. $[3,5,32-36]$ These studies have used strain elastography for the assessment of the MN and found that patients with CTS have a stiffer MN with larger CSA compared to the healthy population. Yoshi et al. (2017), reported significant correlations among NCSs, strain ratio, and CSA in CTS patients. [4]

Among the previous studies, only the study of Martin and Cartwright (2017) that reported no significant difference in the strain ratio between those with CTS and the controls and that the strain ratio did not correlate significantly with the traditional US parameters of CTS. [9]

In addition, Park et al. (2021) reported opposite results regarding the strain ratio.[37] They studied 95 wrists using strain elastography and shear wave elastography (SWE) diagnosed by EDX as CTS. The strain ratio showed no statistically significant difference between CTS patients and healthy controls and there was no significant correlation with CTS severity. However, the elasticity was significantly different between the two groups when evaluated by SWE. [37] MN hardness detected by elastography reflects the histological quantitative changes in the MN. The pathophysiology of CTS is believed to be a combination of increased intracarpal tunnel pressure and ischemic injury of the MN. Increased pressure in the carpal tunnel causes stagnation or blockage of venous outflow and provokes venous congestion and hyperemia, followed by intra-neural edema. Long-term edema causes fibroblast invasion, which results in perineural thickening, nerve fiber changes and the accumulation of scar tissue inside the MN, which lead to increased stiffness of the nerve. [38] In addition, fibrosis of the MN in CTS is not only caused by intraneural edema, but also changes in the oxidative stress and antioxidant defenses could be relevant to fibrosis through the disturbed signaling pattern in the tenosynovium and $\mathrm{MN}$ in patients with CTS. [32]

There are several limitations in our study; sonoelastography requires a meticulous technique in general, regardless of the sonoelastography mode. Various factors such as type of the ultrasound machine, orientation of the probe relative to the ROI, effect of structures adjacent to the ROI can lead to varying results. In our study, tendons were used as a reference point to calculate an ER. This may be a drawback as we assumed that the stiffness of the tendons remains relatively constant from a patient to a patient.

Moreover, in a population sample such our patients and those of other researchers, the relation between the MN stiffness, demographic data and other 
NMUS parameters could not be fully determined. Larger multicenter studies are recommended.

\section{Conclusion}

In CTS, sonoelastography showed valuable correlations with NCSs. Our study confirmed that sonoelastography might have a beneficial and practical value in CTS.

\section{Reference}

[1] F.Kantarci . Median nerve stiffness measurement by shear wave elastography: a potential sonographic method in the diagnosis of carpal tunnel syndrome. European Radiology.vol.24(2),pp.434-440,2014.

[2] Practice parameter for carpal tunnel syndrome (Summary statement).pdf,1993.

[3] O.Ciloglu,and F.F.Gorgulu. Which Ultrasound Parameter Is More Accurate in the Diagnosis of Carpal Tunnel Syndrome: Cross-Sectional Area, Resistive Index, or Strain Ratio? Am J Phys Med Rehabil.vol.99(9),pp.842-846,2020.

[4] Y.Yoshii,W.L.Tung,and T.Ishii, Measurement of Median Nerve Strain and Applied Pressure for the Diagnosis of Carpal Tunnel Syndrome. Ultrasound Med Biol.vol.43(6),pp.1205$1209,2017$.

[5] I.G.Tatar.Carpal tunnel syndrome: elastosonographic strain ratio and crosssectional area evaluation for the diagnosis and disease severity. Med Ultrason.vol.18(3),pp.305-11,2016.

[6] H.Arslan.The efficiency of acoustic radiation force impulse (ARFI) elastography in the diagnosis and staging of carpal tunnel syndrome. J Med Ultrason.vol.45(3),pp.453-459,2018.

[7] Y.Yoshii,C. Zhao, and P.C. Amadio, Recent Advances in Ultrasound Diagnosis of Carpal Tunnel Syndrome. Diagnostics (Basel).vol.10(8),pp.122-150,2020.

[8] C.P.Lin.Utility of Ultrasound Elastography in Evaluation of Carpal Tunnel Syndrome: A Systematic Review and Meta-analysis. Ultrasound Med Biol.vol.45(11),pp.28552865,2019.

[9] M.J.Martin, and M.S. Cartwright, A Pilot Study of Strain Elastography in the Diagnosis of Carpal Tunnel Syndrome. J Clin Neurophysiol.vol.34(2),pp.114-118,2017.

[10]C.K.Jablecki. Practice parameter Electrodiagnostic studies in carpal tunnel syndrome: Report of the American Association of Electrodiagnostic Medicine, American Academy of Neurology, and the American Academy of Physical Medicine and Rehabilitation. Neurology.vol.58(11),pp.1589$1592,2002$.

[11] M.S.Cartwright, and F.O. Walker, Neuromuscular ultrasound in common entrapment neuropathies. Muscle \& nerve.vol.48(5): p. 696-704. 2013

[12] J.D.Bland, A neurophysiological grading scale for carpal tunnel syndrome. Muscle \& Nerve: Official Journal of the American Association of Electrodiagnostic Medicine.vol.23(8),pp.1280$1283,2000$.

[13] M.Sahebari.High-resolution ultrasonography of cross-sectional area of median nerve compared with electro-diagnostic study in carpal-tunnel syndrome.

Rheumatology Research.vol.2(4),pp.127-131,2017.

[14]D.Ažman,P. Hrabač, and V. Demarin, Use of multiple ultrasonographic parameters in confirmation of carpal tunnel syndrome. Journal of Ultrasound in Medicine.vol.37(4),pp.879$889,2018$.

[15]P.Roomizadeh.Ultrasonographic assessment of carpal tunnel syndrome severity: A systematic review and meta-analysis. American journal of physical medicine \& rehabilitation.vol.98(5),pp.373-381,2019.

[16]P.Aggarwal,V. Jirankali, and S.K. Garg, Accuracy of high-resolution ultrasonography in establishing the diagnosis of carpal tunnel syndrome. ANZ journal of surgery.vol.90(6),pp.1057-1061,2020.

[17] T.Torres-Cuenca.Correlation of nerve conduction studies with findings of the ultrasound of the median nerve in patients with carpal tunnel syndrome. Current Medical Imaging.vol.12,pp.140-180,2021.

[18] M.Ratasvuori.Ultrasonography for the diagnosis of carpal tunnel syndrome: correlation of clinical symptoms, cross-sectional areas and electroneuromyography. Journal of Hand Surgery (European Volume).vol.2021,pp.175319,2020 .

[19] H.Sugimoto,N. Miyaji, and T. Ohsawa, Carpal tunnel syndrome: evaluation of median nerve circulation with dynamic contrast-enhanced MR imaging. 466,1994.

[20] L.D.Hobson-Webb.The ultrasonographic wristto-forearm median nerve area ratio in carpal tunnel syndrome. Clin Neurophysiol.vol.119(6),pp.1353-7,2008.

[21] S.Billakota, and L.D. Hobson-Webb, Standard median nerve ultrasound in carpal tunnel syndrome: A retrospective review of 1,021 cases. Clinical neurophysiology practice.vol.12,pp.188-191,2017.

[22] L.H.Visser,M.H. Smidt, and M.L.Lee, Diagnostic value of wrist median nerve cross sectional area versus wrist-to-forearm ratio in carpal tunnel syndrome. Clin Neurophysiol.vol.119(12),pp.2898-2899,2008.

[23] C.B.Gonzalez-Suarez.Diagnostic accuracy of ultrasound parameters in carpal tunnel syndrome: additional criteria for diagnosis. 
Journal of Ultrasound in Medicine.vol.38(11),pp.3043-3052,2019.

[24] I. N.Lo,P.-C. Hsu, and Y. C. Huang, The effect of anti-adhesion gel on median nerve mobility and early clinical outcome in carpal tunnel release. Journal of Hand Surgery (European Volume), .vol.46(3),pp,316-318,2021.

[25] G. y.Park,Usefulness of ultrasound assessment of median nerve mobility in carpal tunnel syndrome.

Acta Radiologica.vol.59(12),pp.1494-1499,2018.

[26] V.J.Schrier.Median nerve transverse mobility and outcome after carpal tunnel release. Ultrasound in medicine \& biology.vol.45(11),pp.2887-2897,2019.

[27] S.Wong.Discriminatory sonographic criteria for the diagnosis of carpal tunnel syndrome. Arthritis \& Rheumatism: Official Journal of the American College of Rheumatology.vol.46(7),pp.1914-1921,2002.

[28] S.C.Roll,J. Case-Smith, and K.D. Evans, Diagnostic accuracy of ultrasonography vs. electromyography in carpal tunnel syndrome: a systematic review of literature. Ultrasound in medicine \& biology.vol.37(10),pp.1539$1553,2011$.

[29] Y. W.Chang.Ratio and difference of the crosssectional area of median nerve to ulnar nerve in diagnosing carpal tunnel syndrome: a case control study. BMC medical imaging.vol.19(1),pp.1-9, 2019.

[30] A.Mallouhi.Predictors of carpal tunnel syndrome: accuracy of gray-scale and color Doppler sonography. AJR Am J Roentgenol.vol.186(5),pp.1240-5,2006.

[31] M.Pertea.Ultrasonography of the Median Nerve in the Carpal Tunnel Syndrome Diagnosis. in E-
Health and Bioengineering Conference (EHB).vol.12,pp.122-150,2019.

[32] G.Orman.Ultrasound elastographic evaluation in the diagnosis of carpal tunnel syndrome: initial findings. Ultrasound Med Biol.vol.39(7),pp.1184-9,2013.

[33] H.Miyamoto.Carpal Tunnel Syndrome: Diagnosis by Means of Median Nerve Elasticity-Improved Diagnostic Accuracy of US with Sonoelastography. Radiology.vol.270(2),pp.481-486,2014.

[34] R.Asadov.The effectiveness of ultrasonography and ultrasonographic elastography in the diagnosis of carpal tunnel syndrome and evaluation of treatment response after steroid injection. Eur J Radiol.vol.108,pp.172176,2018

[35] E.Karatay,O. Turkoglu, and B. Mansiz-Kaplan, Karpal tünel sendromunda median sinir sertliği ölçümlerini kullanarak real-time strain elastografinin tanısal etkinliğinin değerlendirilmesi. Cukurova Medical Journal.vol.45(4),pp.1276-1282,2020.

[36] A.Mahmoud.The Role of Sonoelastography in Diagnosis and Grading of Patients with Carpal Tunnel Syndrome. The Medical Journal of Cairo University.vol.89,pp.2203-2211,2021.

[37] E.J.Park.Comparison of the Diagnostic Performance of Strain Elastography and Shear Wave Elastography for the Diagnosis of Carpal Tunnel Syndrome. J Ultrasound Med.vol.40(5),pp.1011-1021,2021.

[38] M.Cingoz.Evaluation of median nerve by shear wave elastography and diffusion tensor imaging in carpal tunnel syndrome. Eur J Radiol.vol.101,pp.59-64,2018. 